\title{
Structural and Electronic Properties of Pure Ta, TaNO, and TaZrNO with Ab Initio Calculations
}

\author{
T. Chihi, ${ }^{1}$ M. Fatmi, ${ }^{2,3}$ M. Guemmaz, ${ }^{4}$ and J. C. Parlebas ${ }^{5}$ \\ ${ }^{1}$ Laboratory for Elaboration of New Materials and Characterization (LENMC), University of Ferhat Abbas, Setif 19000, Algeria \\ ${ }^{2}$ Research Unit on Emerging Materials (RUEM), University of Ferhat Abbas, Setif 19000, Algeria \\ ${ }^{3}$ Laboratory of Physics and Mechanics of Metallic Materials (LP3M), University of Ferhat Abbas, Setif 19000, Algeria \\ ${ }^{4}$ DAC Laboratory, Faculty of Sciences, University of Ferhat Abbas, Setif 19000, Algeria \\ ${ }^{5}$ IPCMS, UMR 7504 CNRS-UDS, 23 Rue de Loess, 67034 Strasbourg Cedex 2, France
}

Correspondence should be addressed to M. Fatmi, fatmimessaoud@yahoo.fr

Received 8 October 2012; Accepted 15 November 2012

Academic Editors: A. Chrysanthou, Y. Hao, M.-C. Wang, and Y.-d. Wang

Copyright (C) 2012 T. Chihi et al. This is an open access article distributed under the Creative Commons Attribution License, which permits unrestricted use, distribution, and reproduction in any medium, provided the original work is properly cited.

\begin{abstract}
This paper presents the results of self-consistent first-principle calculations for the crystal structure and electronic structure of pure tantalum, TaNO, and TaZrNO within density functional theory (DFT) for the sake of comparison and shows the influence of allowing elements on the interatomic distance and the Fermi level. The large total densities of states (TDOS) value for TaZrNO implies the highest electronic conductivity. The difference in values is due to the $\mathrm{Zr}$ metallic atoms presence in TaZrNO compound. There is a strong interaction between $\mathrm{Ta}$ and $(\mathrm{N}, \mathrm{O})(\mathrm{Ta}-\mathrm{N}=0.39, \mathrm{Ta}-\mathrm{O}=0.21)$ in $\mathrm{TaON}$ compound, and $\mathrm{Zr}$ presence increases this interaction $(\mathrm{Ta}-\mathrm{N}=1.74, \mathrm{Ta}-\mathrm{O}=0.30)$ in $\mathrm{TaZrON}$ compound.
\end{abstract}

\section{Introduction}

The elemental tantalum Ta crystallizes in three crystalline phases, bcc-Ta ( $\alpha$-phase), f.c.c-Ta, and a new phase which is now generally referred to as $\beta$-tantalum. The discoverers of the tetragonal tantalum $\beta$-Ta (a metastable phase), in 1965 are Read and Altman [1]. It has been attracting much interest in most applications because of its high resistivity (170 $210 \mu \Omega \mathrm{cm}$ ) [2-5]. It is preferred for fabricating capacitors and resistors. The chemical stability and robust mechanical properties of Ta make it a particularly desirable material. Numerous crystal structures have been reported for $\beta$-Ta. A tetragonal unit cell Ta was proposed by Read and Altman [1] Das [6] proposed a bcc-based superlattice structure, while Burbank [7] proposed a hexagonal hcp structure and $\beta$ uranium model that was also proposed by Arakcheeva et al. $[8,9]$ on the basis of X-ray diffraction (XRD) study on single crystals of $\beta$-Ta produced through electrolytic crystallization, and in the end, the anomalous f.c.c-Ta structure was observed in very thin films of tantalum $[10,11]$.

On the other hand, nitride formation is common to most transition elements. Many compositional and structural forms exist, with many transition elements forming several different nitride phases. In many of these compounds, nitrogen atoms occupy interstitial lattice sites because they are smaller than the metal atoms. For this reason, they are often referred to as interstitial compounds. Transition metal nitrides are refractory metals that possess technologically useful properties including superconductivity and ultrahigh hardness, and they combine various physical and chemical properties, such as high melting points (around $3000^{\circ} \mathrm{C}$ ). They also possess electronic and magnetic properties that make them useful as electronic and magnetic components and as superconductors [12].

Although monometallic nitrides have been the object of considerable studies [13-15], bimetallic transition metal nitrides have attracted only limited attention. Van Dover et al. [16] investigated the Ternary Transition-Metal Nitride $\mathrm{Y}-\mathrm{Nb}-\mathrm{N}$ and Gd-Cr-N Systems by reactive sputtering, providing evidence for a new superconducting $(\mathrm{Nb}, \mathrm{Y}) \mathrm{N}$ solid solution.

Similarly, the literature on oxynitrides has been scarce. Oxynitrides of transition metals are a new exciting class of materials [17] that possess interesting refractory behaviour, 
higher elastic modulus, and hardness. They also offer great potential for their optical properties and recently have received much attention because of their potential use as pigment materials [18]. It has been known that oxygen atoms can substitute nitrogen atoms in monometallic nitrides due to the similarity in their radius. In many of the oxynitrides compounds, the $\mathrm{N}$ and $\mathrm{O}$ atoms are found in interstitial lattice positions in between the metal atoms. For this reason, their phases can exist over broad composition ranges with appreciable vacancy concentrations (both metal and nonmetal) and their physical properties are quite sensitive to composition. Yashima et al. [19] investigate neutron diffraction for confirmation of anion ordering and synchrotron powder diffraction for high-precision analysis of the crystal structure and electron density of an active TaON photocatalyst sample under visible-light excitation. Yashima et al. [20] employ the density functional theory (DFT) for theoretical calculations of the electron density distribution and partial density of states of $\mathrm{TaON}$ compound.

Our primary aim was therefore to present the results of a theoretical investigation of the structural and electronics of metastable $\beta$-Ta, bcc-Ta, and f.c.c-Ta. The bcc-Ta ((f.c.c$\mathrm{Ta})$ ) structures are cubic; the space group is $\mathrm{Im}-3 \mathrm{~m}$ (no.229) (Fm-3 m (no.225)) with two (four) formula units per unit cell, the metastable $\beta$-Ta $(\beta$-uranium, Distorted A15, and Hexagonal) structures are (tetragonal $\mathrm{P} 4_{2} / \mathrm{mnm}$ (no.136), distorted $\mathrm{Pm}(-3) \mathrm{m}$ (no.223), and hexagonal $\mathrm{P}_{3} / \mathrm{mmc}$ (no.194)). The bimetal (monometal) transition metal oxynitride TaZrNO (TaNO) structures are hexagonal (cubic), and the space group is $P \overline{6} m 2$ (no.187) ( $F \overline{4} 3 m$ (no.216)) with one (four) formula unit(s) per unit cell. Until now, there has been no report on the electronic properties of pure tantalum, TaNO, and TaZrNO.

This paper presents the results of self-consistent firstprinciples calculations for the crystal structure and electronic structure of pure tantalum, TaNO, and TaZrNO within DFT for the sake of comparison and shows the influence of allowing elements on the interatomic distance and the Fermi level.

The paper is organized as follows. The computational method is described in Section 2. In Section 3, the results are presented and compared with available experimental and theoretical data. Conclusion is given in Section 4.

\section{Computational Method}

All calculations were performed by using the CASTEP (Cambridge Serial Total Energy Package) simulation program [21] that solves the Schrodinger-like Kohn-Sham equations according to the formalism of the density functional theory (DFT) [22, 23]. We used the Generalized Gradient Approximation (GGA) and a Perdew-Burke-Ernzerhof (PBE) scheme [24] for handling the electronic exchangecorrelation potential energy. Also, the pseudopotentials constructed using the ab initio norm conserving scheme describe the valence electron interaction with the atomic core, in which the Ta $\left(4 f^{14} 5 d^{3} 6 s^{2}\right), \operatorname{Zr}\left(4 d^{2} 5 s^{2}\right), \mathrm{N}\left(2 s^{2} 2 p^{3}\right)$, and $\mathrm{O}\left(2 s^{2} 2 p^{4}\right)$ orbitals are treated as valence electrons. Using

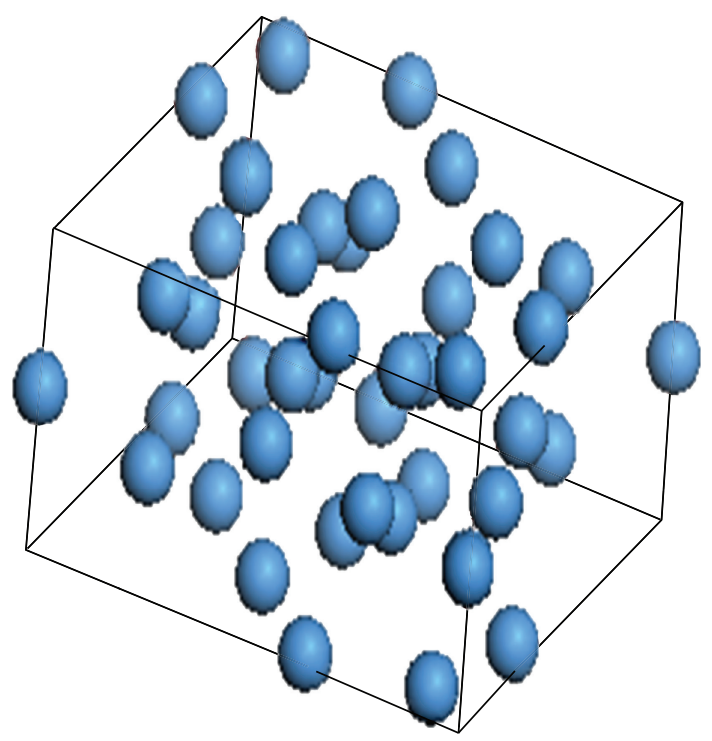

Figure 1: (Ta) $\beta$-uranium structure.

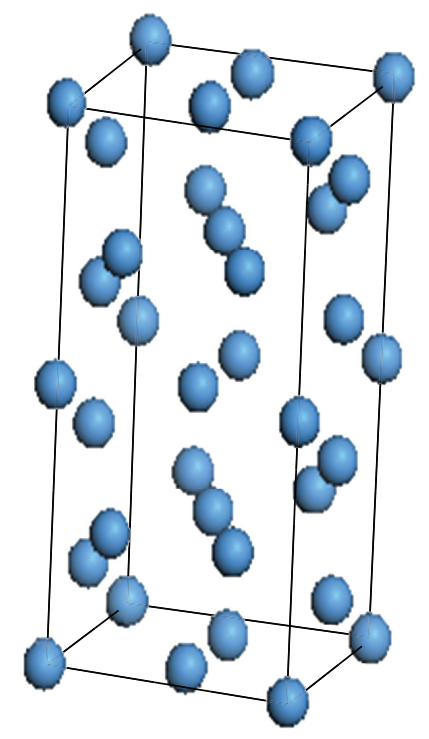

Figure 2: (Ta) Distorted A15 $\beta$-structure.

for all structures high cut-off energy $(280 \mathrm{eV})$ even at the price of spending long computational time is the condition to obtain accurate results. Brillouin zone (BZ) sampling is carried out using a $6 \times 6 \times 6$ Monkhorst-Pack mesh set [25]. For Ta (distorted A15) and hexagonal (type Cd or $\mathrm{Zn}$ ) structures, the BZ sampling is carried out using a $10 \times 10 \times 6$ Monkhorst-Pack mesh and a cut-off energy of $(280 \mathrm{eV})$. Atomic positions are relaxed and optimized within a density mixing scheme, based on a Conjugate Gradient (CG) method for eigenvalues minimization. Actually, the equilibrium lattice parameter is determined from a structural optimization, using the Broyden-Fletcher-Goldfarb-Shenno (BFGS) minimization technique. This technique provides a fast way of finding the lowest energy structure, with the 


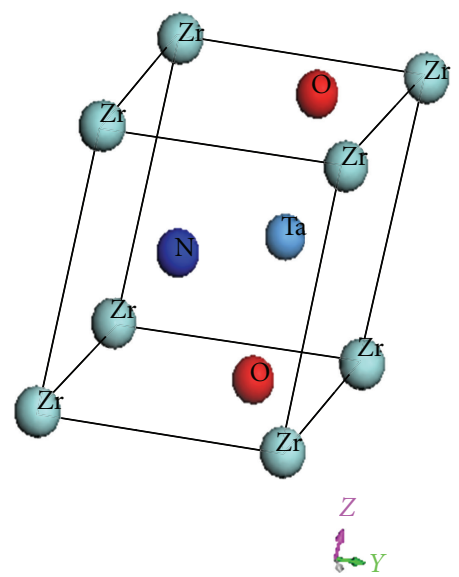

Figure 3: TaZrNO structure.

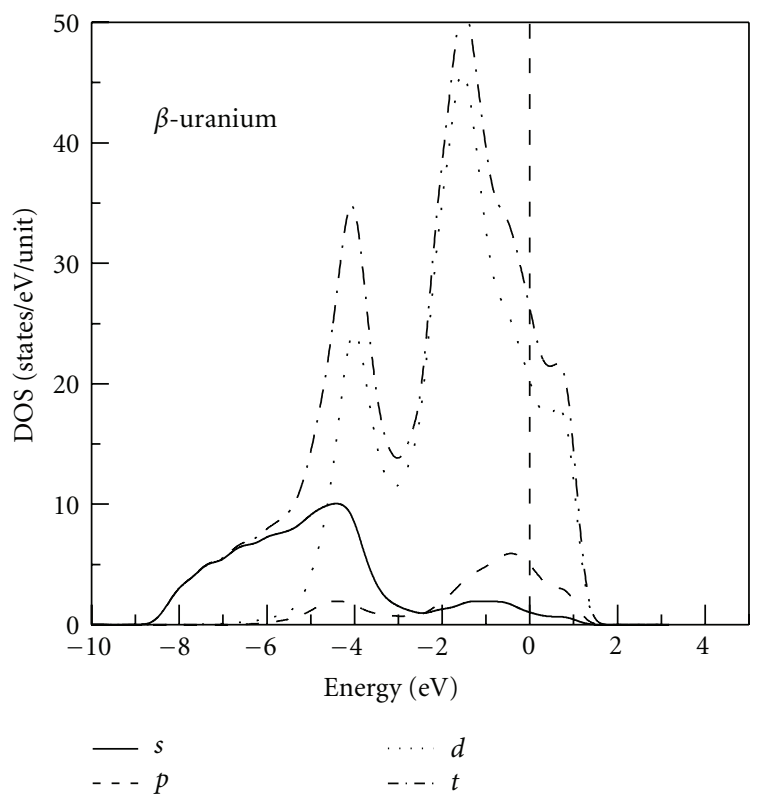

Figure 4: Total and partial densities of states for $\beta$-Ta ( $\beta$-uranium).

following thresholds for converged structures: (i) energy change per atom less than $2 \times 10^{-5} \mathrm{eV}$, (ii) residual force less than $0.05 \mathrm{eV} / \AA$, (iii) atom displacement during geometry optimization less than $0.002 \AA$, and (iv) maximum stress within $0.1 \mathrm{GPa}$. The crystal structures of Ta: $\beta$-uranium, Ta: Distorted A15 $\beta$-structure, and TaZrNO structure are given in (Figures 1, 2, and 3).

\section{Results and Discussion}

3.1. Structural Properties. Results for the lattice parameter $a, c$ and $c / a$ are reported (Table 1) and compared with experimental and theoretical calculations. Our calculated GGA values for $a$ for pure tantalum are in good agreement with the experimental data $[1,3,7]$. We have obtained the same value for $a$ of TaNO as experimental [26] one. For TaZrNO, our computed lattice constant $(a)$ is bigger

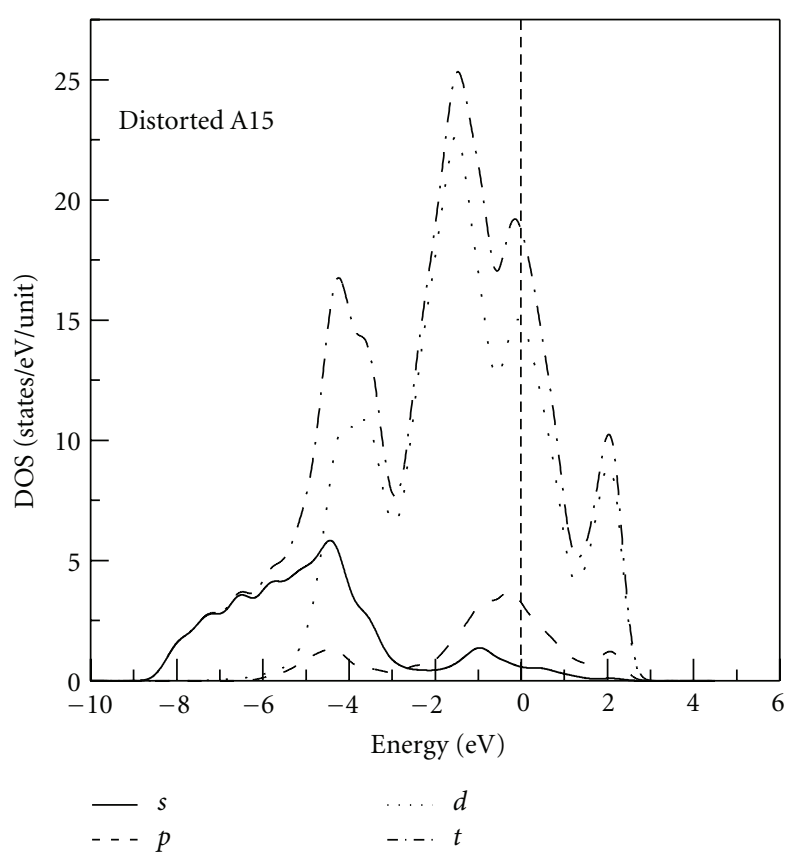

FIgure 5: Total and partial densities of states for $\beta$-Ta (distorted A15).

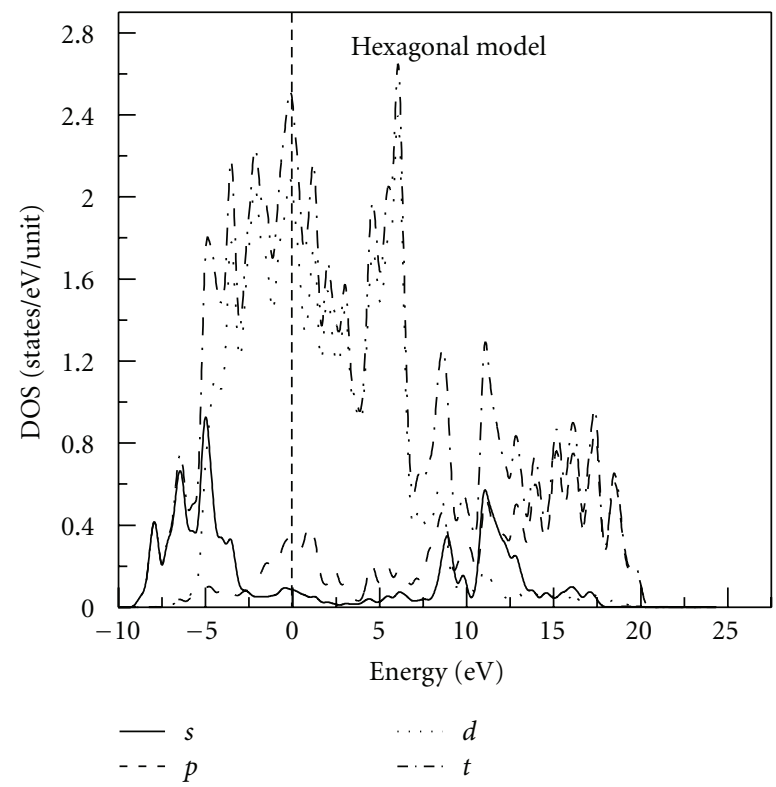

FIgURE 6: Total and partial densities of states for $\beta$-Ta (hexagonal model).

than the experimental one, within 7.4\% [27]. Also, for both structures, the calculated lattice parameter ratio $c / a(0.523$, 1.978 , and 1.792 for $0020 \beta$-uranium, distorted A15, and hexagonal, resp.) is in reasonable accord with the previously considered data $(0.531,1.860$, and $1.890,[1,7,28-31]$ and 1.098 for TaZrNO that is also in reasonable accord with of 1.064) [27]. In order to attempt an understanding the interatomic distances of the various compounds, we have found excellent agreement between our calculated 


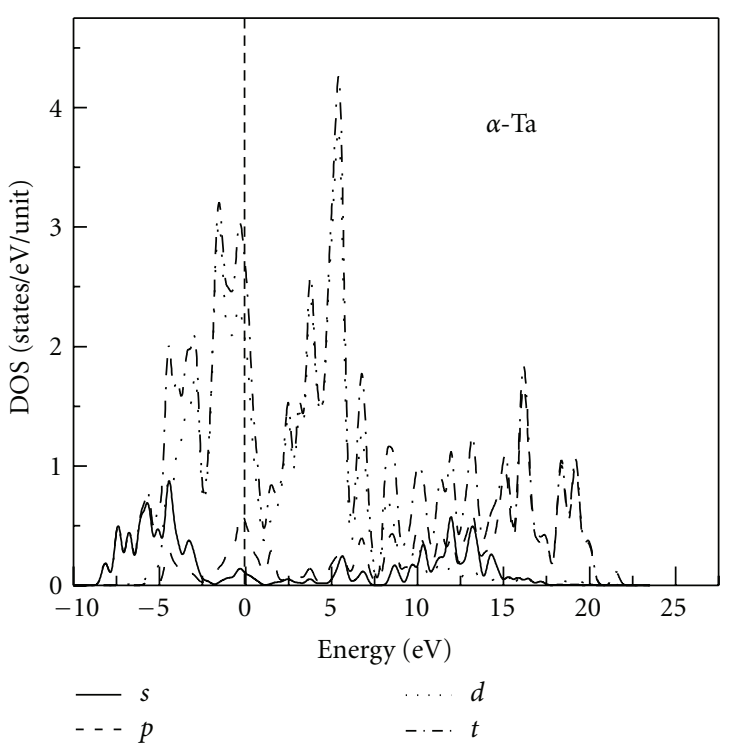

Figure 7: Total and partial densities of states for $\alpha$-Ta.

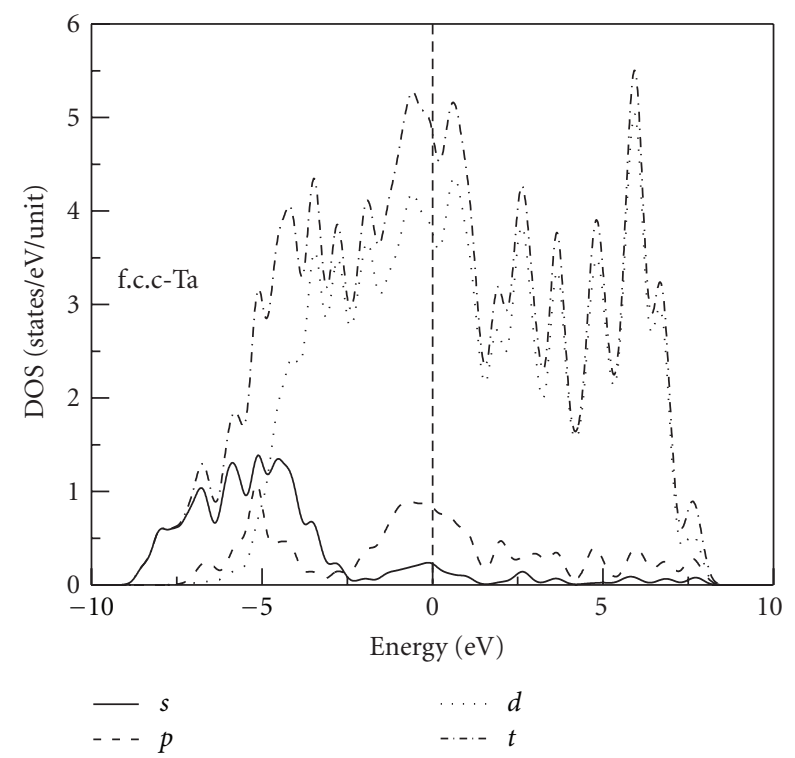

FIgURE 8: Total and partial densities of states for f.c.c-Ta.

and available experiment interatomic distances for different phases of pure tantalum. The results of distances are reported in Table 1.

3.2. Electronic Structures. Density of states and electronic band structure often provide sufficient information for a thorough characterization of the electronic properties of the material. Total density of states (DOS) of all $\beta$-Ta (metastable phase), $\alpha$-Ta, and f.c.c-Ta structures were calculated in order to understand differences in the chemical bonding between them. The DOS for $\beta$-Ta structure, shown in Figures 4 and 5, is concentrated in two peaks. The low energy peak, between -9 and $-3 \mathrm{eV}$ (the zero of energy is taken at the Fermi energy $E_{f}$ ), is comprised mostly of $s$ and $d$ states. The other broad peak, lying at and above $E_{f}$, has $d$ character with small admixture of $p$ characters and is responsible for the $\mathrm{N}\left(E_{f}\right)$ of 26.024 and 18.268 states/eV $\AA^{3}$ for $\beta$-uranium and distorted A15 models, respectively. For the hexagonal model, the DOS is mainly due to the $d$ states, with small $s$ characters at around -5 and $10 \mathrm{eV}$ (Figure 6). Figures 7 and 8 shows respectively the density of states for $\alpha$-Ta and fcc-Ta that are mainly dominated by $d$ states and little $s$ contributions especially around $-5 \mathrm{eV}$. Both structures are metallic because of the finite DOS at the Fermi level $\left(E_{f}\right)$. The DOS values at $E_{f}$ of all structures are shown in Table 2. Let us mention a large DOS at the Fermi level; this implies a high electronic conductivity and various applications to electronic conductors. Figures 9 and 10 show the total density of states (TDOS) and partial density of states (PDOS) for TaNO and TaZrNO compounds. The TDOS of TaNO (TaZrNO) can be mainly distributed in the following three energy regions in the valence bonding region.

(1) In the region one from approximately $-26 \mathrm{eV}$ to $-23 \mathrm{eV}(-25 \mathrm{eV}$ to $-22 \mathrm{eV})$. In this region, the DOS mainly consists of $s$ orbitals of $\mathrm{O}$, together with a small portion of $s$ orbitals of $\mathrm{Ta}(s$ orbitals of $\mathrm{O}$, together with a small portion of $s$ orbitals of Ta and $\mathrm{Zr}$ ). These states represent the electronic core structures;

(2) In region two, it is from $-18.0 \mathrm{eV}$ to $-16.0 \mathrm{eV}$ $(-19 \mathrm{eV}$ to $-17 \mathrm{eV})$. In this region, the DOS is contributed mainly from the hybridized $s$ orbitals of $\mathrm{N}, d$ orbitals of Ta, together with a small portion of $s$ orbitals of Ta for TaNO, and the same behavior is observed for TaZrNO compounds;

(3) In region three; it is from $-14 \mathrm{eV}$ to $0 \mathrm{eV}(-12 \mathrm{eV}$ to $0 \mathrm{eV})$. This is due to the $p-\mathrm{O}, p-\mathrm{N}$, and $d$-Ta orbitals ( $p-\mathrm{O}, p-\mathrm{N}, d-\mathrm{Ta}$, and $d-\mathrm{Zr}$ with a small portion of $s-$ Ta and $s-\mathrm{Zr}$ contributions). Above the Fermi energy level, the DOS is predominated by $d-\mathrm{Ta}$ and $p-\mathrm{N}$ orbitals with a small portion of $p$-O contribution $(d$ $\mathrm{Ta}, d-\mathrm{Zr}, p-\mathrm{N}$, and $p-\mathrm{O}$ orbitals with a small portion of $p-\mathrm{Ta}$ and $p-\mathrm{Zr}$ contributions), corresponding to the electronic anti-bonding states. The two TDOS have some similarities, however, in Figures 9 and 10.

TDOS at the Fermi level is 1.493 for TaNO.

TDOS at the Fermi level is 2.010 for TaZrNO, and the large value for TaZrNO implies the highest electronic conductivity.

The difference in values is due to the $\mathrm{Zr}$ metallic atoms presence in TaZrNO compound.

The pure tantalum has the highest $\left(E_{f}\right)$, and generally speaking, the smaller the $\left(E_{f}\right)$ is, the unstable the compound will be.

3.2.1. Bond Orders between Atoms. Bond order is the overlap population of electrons between atoms, and this is a measure of the strength of the covalent bond between atoms. If the overlap population is positive $(+)$, a bonding-type interaction is operating between atom, whereas if it is negative $(-)$, an antibonding-type interaction is dominant between 


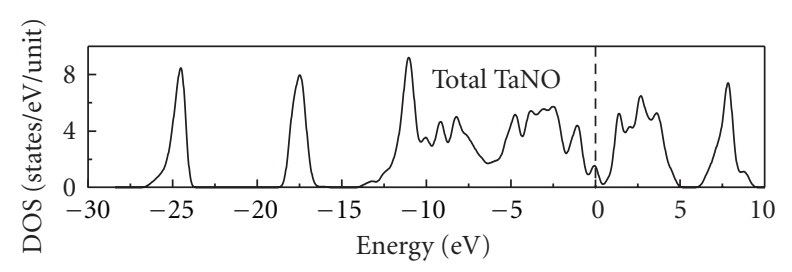

(a)

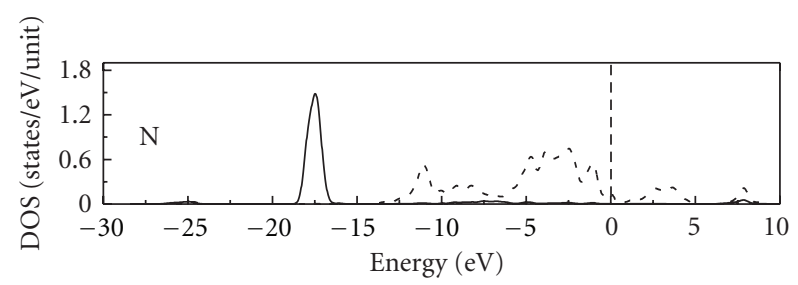

$-s$
$--p$

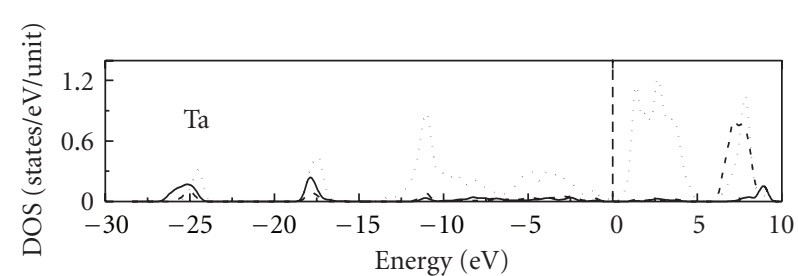

$-s$
$--p$

(b)

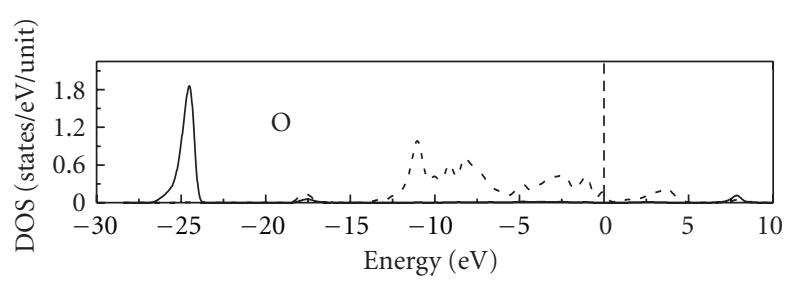

$-s$

(c)

(d)

FIgURE 9: Total and partial densities of states for TaNO.

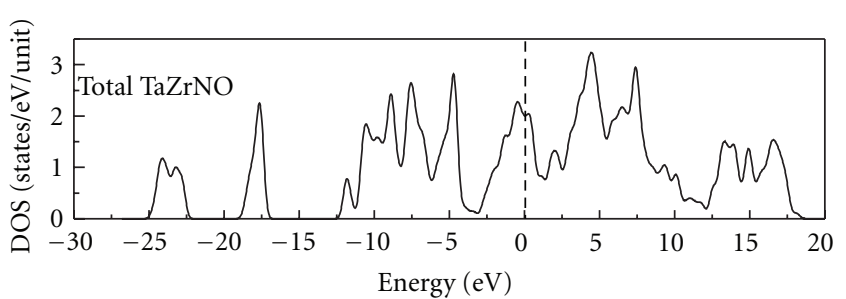

(a)

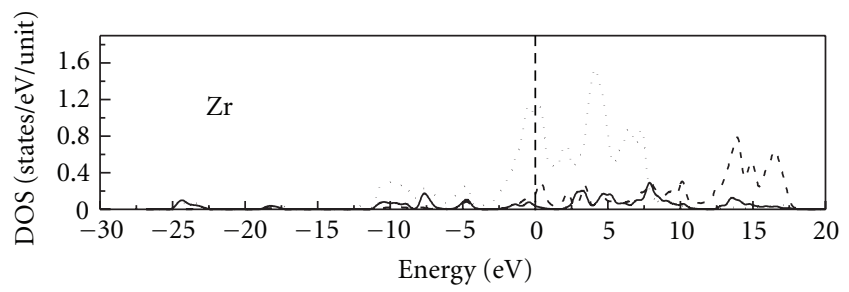

$-s$
$--p$

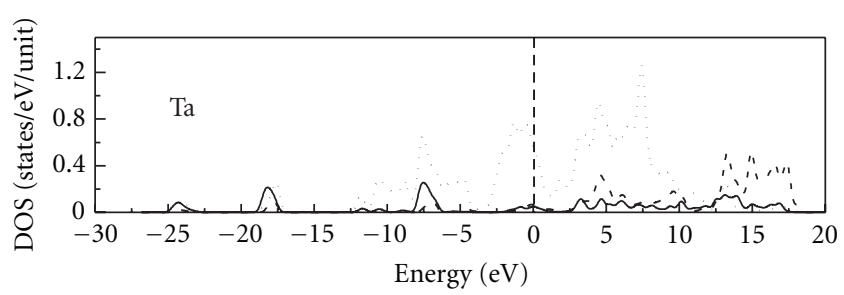

$-s$

(b)

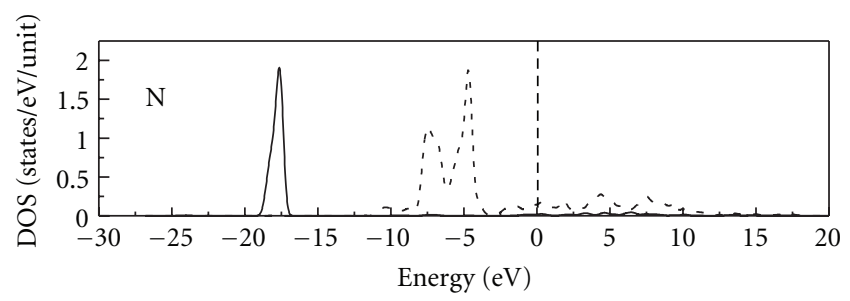

$-s$

(c)

(d)

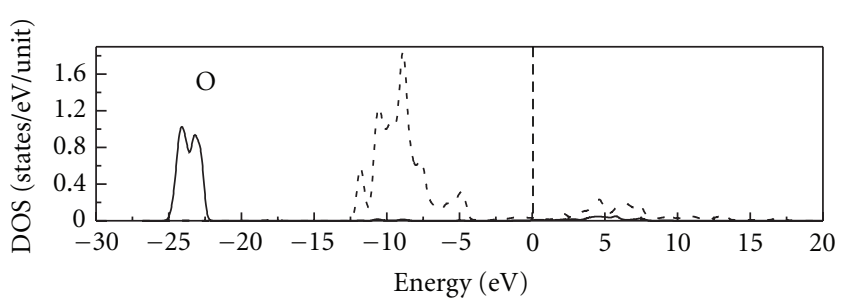

$\ldots s$

(e)

Figure 10: Total and partial densities of states for TaZrNO. 
TABle 1: Lattice constant, shortest Ta-Ta, density, and atomic/unit cell of the all metastable phase.

\begin{tabular}{|c|c|c|c|c|c|c|}
\hline Materials & $\begin{array}{c}\text { Lattice constant } \\
a_{0}(\AA)\end{array}$ & $c / a$ & $\begin{array}{c}\text { Shortest Ta-Ta } \\
(\AA)\end{array}$ & $\begin{array}{l}\text { Density } \\
\left(\mathrm{g} / \mathrm{cm}^{-3}\right)\end{array}$ & Atom/unit cell & Reference \\
\hline \multirow{2}{*}{$\beta$-uranium } & 9.9345 & 0.523 & 2.608 & 17.582 & 30 & \multirow{2}{*}[28,29]{} \\
\hline & 10.194 & 0.531 & 2.650 & 16.330 & 30 & \\
\hline \multirow{2}{*}{ Distorted A15 } & 5.1551 & 1.978 & 2.550 & 17.736 & 16 & \multirow{2}{*}[1,30,31]{} \\
\hline & 5.3400 & 1.860 & 1.890 & 16.960 & 16 & \\
\hline \multirow{2}{*}{ Hexagonal } & 2.804 & 1.792 & 2.805 & 17.605 & 2 & \multirow{2}{*}[7]{} \\
\hline & 2.830 & 1.890 & 2.830 & 16.230 & 2 & \\
\hline \multirow{2}{*}{$\alpha-\mathrm{Ta}$} & 3.217 & - & 2.786 & 18.050 & 2 & \multirow{2}{*}{ [3] } \\
\hline & 3.31 & - & 2.860 & 16.550 & 2 & \\
\hline \multirow{2}{*}{ f.c.c-Ta } & 4.108 & - & 2.905 & 17.329 & 4 & \multirow{4}{*}{$\begin{array}{c}{[11-31]} \\
{[26]}\end{array}$} \\
\hline & 4.390 & - & 3.100 & 14.21 & 4 & \\
\hline \multirow{2}{*}{ TaNO } & 4.827 & - & 3.031 & 17.798 & 12 & \\
\hline & 4.827 & - & - & - & - & \\
\hline \multirow{2}{*}{ TaZrNO } & 3.373 & 1.098 & 3.707 & 13.733 & 4 & \multirow[t]{2}{*}[27]{} \\
\hline & 3.645 & 1.064 & - & - & - & \\
\hline
\end{tabular}

TABLe 2: Fermi level $E_{f}$ of the all metastable phase.

\begin{tabular}{cccccccc}
\hline & $\beta$-uranium & Distorted A15 & Hexagonal & $\alpha$-Ta & f.c.c-Ta & TaNO & TaZrNO \\
\hline$E_{f}$ & 26.272 & 18.268 & 2.418 & 2.579 & 4.704 & 1.493 & 2.010 \\
\hline
\end{tabular}

atoms. It is apparent that the bonding-type interactions are operating between the metal $\mathrm{M}(\mathrm{M}=\mathrm{Ta}, \mathrm{Zr})(4 d$ and $5 d)$ and the nonmetal $(\mathrm{N}, \mathrm{O}) 3 d$ electrons. Thus, there is a strong interaction between $\mathrm{Ta}$ and $(\mathrm{N}, \mathrm{O})(\mathrm{Ta}--\mathrm{N}=0.39$, $\mathrm{Ta}--\mathrm{O}=0.21)$ in $\mathrm{TaON}$ compound, and $\mathrm{Zr}$ presence increases this interaction $(\mathrm{Ta}--\mathrm{N}=1.74, \mathrm{Ta}--\mathrm{O}=0.30)$ in TaZrON compound.

\section{Summary and Conclusion}

Using the first principles based on the DFT, we studied the total (partial) density of states TDOS (PDOS) of pure tantalum, TaNO, and TaZrNO. The large value for TaZrNO implies the highest electronic conductivity. The difference in values is due to the $\mathrm{Zr}$ metallic atoms presence in TaZrNO compound. A bonding-type interaction is operating between atoms, and thus there is a strong interaction between Ta and $(\mathrm{N}, \mathrm{O})(\mathrm{Ta}--\mathrm{N}=0.39, \mathrm{Ta}--\mathrm{O}=0.21)$ in $\mathrm{TaON}$ compound, and $\mathrm{Zr}$ presence increases this interaction ( $\mathrm{Ta}-$ $-\mathrm{N}=1.74, \mathrm{Ta}--\mathrm{O}=0.30)$ in $\mathrm{TaZrON}$ compound.

\section{References}

[1] M. H. Read and C. Altman, "A new structure in tantalum thin films," Applied Physics Letters, vol. 7, no. 3, article 51, 2 pages, 1965.

[2] N. Schwartz, W. A. Reed, P. Polash, and M. H. Read, "Temperature coefficient of resistance of beta-tantalum films and mixtures with b.c.c.-tantalum," Thin Solid Films, vol. 14, no. 2, pp. 333-346, 1972.

[3] P. N. Baker, "Preparation and properties of tantalum thin films," Thin Solid Films, vol. 14, no. 1, pp. 3-25, 1972.
[4] A. Schauer and M. Roschy, "R.F. sputtered $\beta$-tantalum and b.c.c. tantalum films," Thin Solid Films, vol. 12, no. 2, pp. 313317, 1972.

[5] Y. Chouan and D. Collobert, "Plasma influence in tantalum sputtering," Journal of Applied Physics, vol. 48, no. 6, pp. 22742279, 1977.

[6] G. Das, "A new structure of sputtered tantalum," Thin Solid Films, vol. 12, no. 2, pp. 305-311, 1972.

[7] R. D. Burbank, "X-ray study of $\beta$-tantalum," Journal of Applied Crystallography, vol. 6, no. 3, pp. 217-224, 1973.

[8] A. Arakcheeva, G. Chapuis, and V. Grinevitch, "The selfhosting structure of $\beta$-Ta," Acta Crystallographica Section B, vol. 58 , no. 1, pp. 1-7, 2002.

[9] A. Arakcheeva, G. Chapuis, H. Birkedal, P. Pattison, and V. Grinevitch, "The commensurate composite $\sigma$-structure of $\beta$ tantalum," Acta Crystallographica Section B, vol. 59, no. 3, pp. 324-336, 2003.

[10] R. B. Marcus and S. Quingley, "Formation of f.c.c., b.c.c. and $\beta$-tantalum films by evaporation," Thin Solid Films, vol. 2, no. 5-6, pp. 467-477, 1968.

[11] P. N. Dendigh and R. B. Marcus, "Structure of very thin tantalum and molybdenum films," Journal of Applied Physics, vol. 37, no. 12, article 4325, 6 pages, 1966.

[12] T. H. Geballe, B. T. Matthias, J. P. Remeika et al., Physics (Long Island City, N.Y.), vol. 2, p. 293, 1966.

[13] D. A. Papaconstantopoulos, W. E. Pickett, B. M. Klein, and L. L. Boyer, "Electronic properties of transition-metal nitrides: the group-V and group-VI nitrides $\mathrm{VN}, \mathrm{NbN}, \mathrm{TaN}, \mathrm{CrN}$, MoN, and WN," Physical Review B, vol. 31, no. 2, pp. 752-761, 1985.

[14] C. Stampfl, W. Mannstadt, R. Asahi, and A. J. Freeman, "Electronic structure and physical properties of early transition metal mononitrides: density-functional theory LDA, GGA, and screened-exchange LDA FLAPW calculations," Physical Review B, vol. 63, no. 15, Article ID 155106, 11 pages, 2001. 
[15] P. Ojha, M. Aynyas, and S. P. Sanyal, "Pressure-induced structural phase transformation and elastic properties of transition metal mononitrides," Journal of Physics and Chemistry of Solids, vol. 68, no. 2, pp. 148-152, 2007.

[16] R. B. van Dover, B. Hessen, D. Werder, C. H. Chen, and R. J. Felder, "Investigation of ternary transition-metal nitride systems by reactive cosputtering," Chemistry of Materials, vol. 5, no. 1, pp. 32-35, 1993.

[17] T. Das, "Oxynitride glasses - an overview," Bulletin of Material Science, vol. 23, no. 6, pp. 499-5047, 2000.

[18] E. Guenther and M. Jansen, "Optical properties of $\mathrm{Ta}_{(3-x)}$ $\mathrm{Zr}_{(x)} \mathrm{N}_{(5-x)} \mathrm{O}_{(x)}$ semiconductor pigments," Materials Research Bulletin, vol. 36, no. 7-8, pp. 1399-1405, 2001.

[19] M. Yashima, Y. Lee, and K. Domen, "Crystal structure of tantalum oxynitride $\mathrm{TaON}$, a visible light responsive photocatalyst," Experimental Reports, vol. 15, no. 395, 2008.

[20] M. Yashima, Y. Lee, and K. Domen, "Crystal structure and electron density of tantalum oxynitride, a visible light responsive photocatalyst," Chemistry of Materials, vol. 19, no. 3, pp. 588-593, 2007.

[21] M. D. Segall, P. J. D. Lindan, M. J. Probert et al., "Firstprinciples simulation: ideas, illustrations and the CASTEP code," Journal of Physics Condensed Matter, vol. 14, no. 11, pp. 2717-2744, 2002.

[22] P. Hohenberg and W. Kohn, "Inhomogeneous electron gas," Physical Review, vol. 136, no. 3, pp. B864-B871, 1964.

[23] W. Kohn and L. J. Sham, "Self-consistent equations including exchange and correlation effects," Physical Review, vol. 140, no. 4, pp. A1133-A1138, 1965.

[24] J. P. Perdew, S. Burke, and M. Ernzerhof, "Generalized gradient approximation made simple [Phys. Rev. Lett. 77, 3865 (1996)]," Physical Review Letters, vol. 78, no. 7, article 1396, 1997.

[25] H. J. Monkhorst and J. D. Pack, "Special points for Brillouinzone integrations," Physical Review B, vol. 13, no. 12, pp. 5188$5192,1976$.

[26] H. Wolff, H. Schilling, M. Lerch, and R. Dronskowski, "A density-functional and molecular-dynamics study on the physical properties of yttrium-doped tantalum oxynitride," Journal of Solid State Chemistry, vol. 179, no. 8, pp. 2265-2270, 2006.

[27] N. Schonberg, "The structure of the metallic quaternary phase ZrTaNO," Acta Chemica Scandinavica, vol. 8, pp. 627-629, 1954.

[28] P. T. Moseley and C. J. Seabrook, "The crystal structure of $\beta$ tantalum," Acta Cryst B, vol. 29, no. 5, pp. 1170-1171, 1973.

[29] A. C. Lawson and C. R. Olsen, "Structure of $\beta$-uranium," Acta Cryst B, vol. 44, no. 2, pp. 89-96, 1988.

[30] P. Catania, R. A. Roy, and J. J. Cuomo, "Phase formation and microstructure changes in tantalum thin films induced by bias sputtering," Journal of Applied Physics, vol. 74, no. 2, pp. 10081014, 1993.

[31] D. Mills, Journal of the CanadianCeramic Society, vol. 35, p. 48, 1966. 

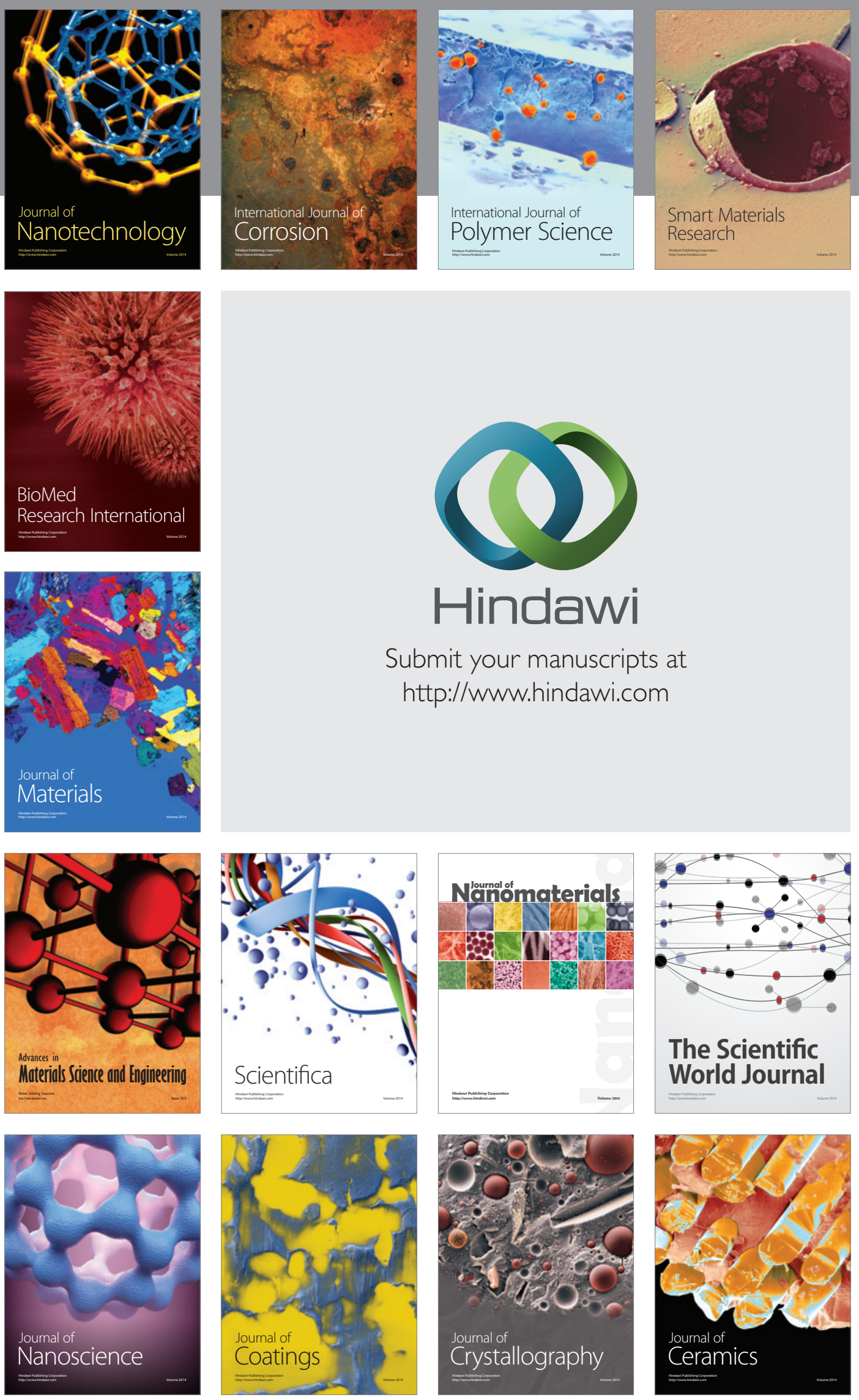

The Scientific World Journal

Submit your manuscripts at

http://www.hindawi.com

\section{World Journal}

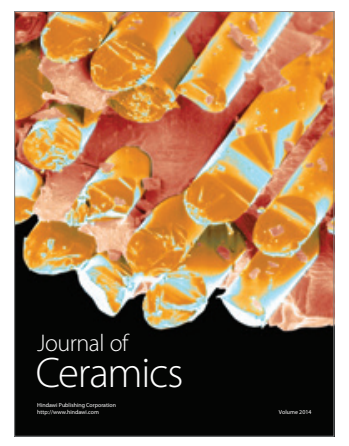

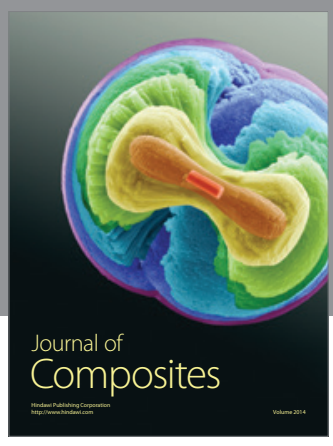
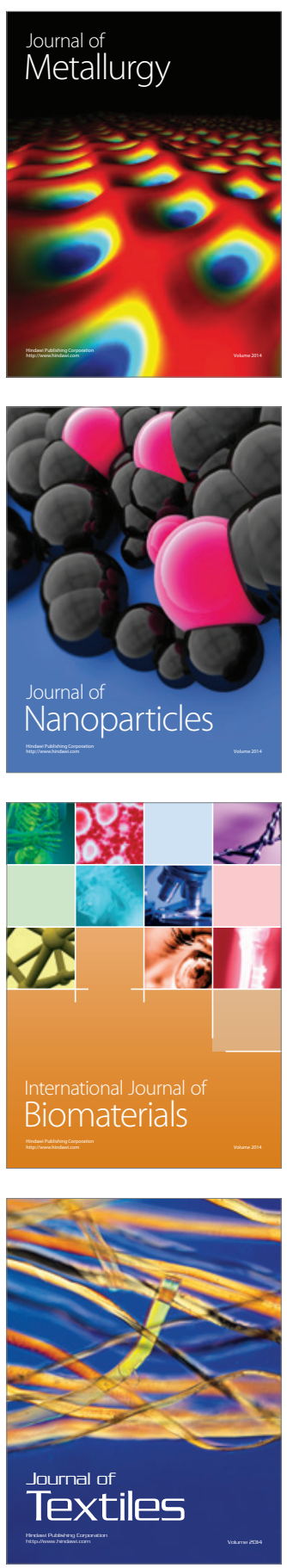